\title{
Jeder vierte alte Mensch hier hat Typ-2-Diabetes
}

Fragestellung: Wie hoch sind Prävalenz und Inzidenz von Diabetes mellitus in Deutschland?

Hintergrund: Überall werden die Zahlen der wachsenden Prävalenz und Inzidenz des Diabetes mellitus als Argument für Aktivitäten zur besseren Behandlung und Prävention genutzt. Hört man aber Vorträge von deutschen Forschern, schwankt die Prävalenz zum Diabetes in Deutschland von $6 \%-12 \%$. Was ist richtig? Leider gibt es keine großen Studien dazu. Aus kleineren Kohortenstudien rechnet man die Zahlen hoch, aber mit sehr verschiedenen Ergebnissen. Andere Daten zu Deutschland stammen aus dem Diabetes-Atlas der International Diabetes Federation, der aber auch die genannten Kohortenstudien genutzt hat. Krankenkassen haben, v. a. durch die Disease-Management-Programme, einen guten Überblick über die Morbidität ihrer Versichertenstruktur. Bisher war es nur kaum möglich, diese Daten wissenschaftlich zu analysieren. Düsseldorfer Forscher um Tamayo und Rathmann hatten nun die Möglichkeit, 65 Mio. Versichertendaten der Gesetzlichen Krankenkassen (GKV) auszuwerten.

Originalie

Tamayo T, Brinks R, Hoyer A et al. The Prevalence and Incidence of Diabetes in Germany. Dtsch Arztebl Int. $2016 \mathrm{Mar}$ 18;113(11):177-82
Patienten u. Methoden: Es wurden die ICT-kodierten Diabetesdiagnosen aus dem ambulanten und stationären Sektor zur Definition eines Diabetes herangezogen. Das war möglich, da ein Gesetz vom 22.12.2011 Vorschriften zur Datentransparenz bei den Krankenkassen initiierte. Dieses sollte vom Deutschen Institut für Medizinische Dokumentation und Information (DIMDI) umgesetzt werden. Einbezogen wurden 65,6 Millionen Patientendaten, die 2009 über alle vier Quartale bei einer GKV versichert waren.

Ausgeschlossen wurden z.B. Patienten, die im Berichtszeitraum verstorben oder in private Krankenkassen gewechselt sind. Die Daten von 2009 und 2010 wurden verglichen und die Prävalenz der Erkrankung in der Gesamtstichprobe bestimmt. Die Inzidenz wurde mithilfe der Prävalenzänderung zwischen den beiden Jahren und der zu erwartenden Mortalität bei Erkrankten und Nichterkrankten berechnet.

Ergebnisse: 6,4 M von 65,6 Mio. Menschen hatten im Jahr 2009 Diabetes. 2010 waren es 6,7 von 64,9 Mio. GKV-Versicherten. Das entspricht einer alters- und geschlechtsstandardisierten Prävalenz von 9,7\% 2009 und 9,9\% 2010. Von Typ-2-Diabetes waren 2009 4,6 Mio. und 2010 4,7 Mio. Versicherte betroffen. Adjustiert man diese Daten, können wir schlussfolgern, dass in diesem Zeitraum 5,8 Mio. Typ-2-Diabetiker in Deutschland lebten. Die Prävalenz und Inzidenz stieg zwischen dem 15. und 79. Lebensjahr stark an. Die Inzidenz erreicht um das 85. Lebensjahr die höchste Rate mit 24-29 Neuerkrankungen/1000 Patientenjahre.

Schlussfolgerungen: Jeder Vierte in Deutschland ab 80 Jahre ist an Typ-2-Diabetes erkrankt. Durch die Analyse der GKVDaten ist es erstmals möglich, die Daten aus den bisherigen Kohortenstudien in Deutschland zu validieren und genauere Angaben zur Prävalenz und Inzidenz des Diabetes zu erzeugen.

\section{- Kommentar von Prof. Dr. med. Peter Schwarz}

\section{Deutschland raubt sich sehr viel Arbeitskraft und Lebensqualität}

Das ist spannend - aber man weiß nicht so genau wie man die Daten einordnen soll. Es war eine Totalerhebung eines großen Kollektivs der GKV-Versicherten mit solider Methodik. Die Ergebnisse liegen eher am oberen Rand dessen, was diskutiert wurde. V.a. für ältere Patienten bietet diese Studie erstmalig verlässliche Aussagen. Alle Trends, die wir vom Diabetes bisher kennen, sind bestätigt. Es betrifft häufiger Männer und Ältere, und je älter Frauen werden, desto schneller nähern sie sich dem Risiko beim Mann an. Was machen wir jetzt damit? Sicher fehlen die Patienten, die im Berichtszeitraum gestorben sind und jene, die in die private KV gewechselt sind. Vermutlich hat dies aber keinen signifikanten Einfluss. Die Daten sollten für alle von uns Ansporn sein, besser zu behandeln und zu überlegen, wie mit Risikopersonen, die vermutlich mehr sind als Diabetiker, präventiv umgegangen werden kann. Es geht hier um etwa $10 \%$ der Bevölkerung. Schaut man sich andere Bereiche an, wo 10\% der Bevölkerung von einem Nachteil betroffen sind, agieren Regierungen sehr schnell. Bringt man die hohe Prävalenz mit den Daten der OECD zur Beeinträchtigung der Arbeitsfähigkeit sozialer Teilhabe und Lebensqualität durch Diabetes zusammen, raubt sich Deutschland damit sehr viel Arbeitskraft und Lebensqualität. Das sollte Motivation genug sein neue Maßnahmen und „Produkte“ zur Prävention zu entwickeln. Es wäre wünschenswert, dass eine weitere Untersuchung in 10 Jahren niedrigere Zahlen ergibt.

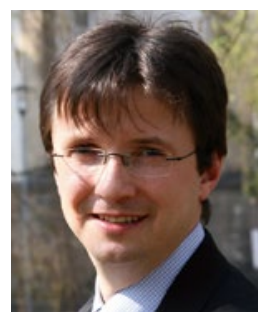

Prof. Dr. med. Peter Schwarz, MBA

Department of Medicine III

Prevention and Care of Diabetes

University of Dresden

Fetscherstr. 74, 01307 Dresden

peter.schwarz@uniklinikum-dresden.de 